(2) Open Access Full Text Article

\title{
Apatinib treatment combined with chemotherapy for advanced epithelial ovarian cancer: a case report
}

This article was published in the following Dove Press journal:

OncoTargets and Therapy

13 March 2017

Number of times this article has been viewed

\author{
Linghui Deng \\ Yue Wang \\ Wenbin Lu \\ Qian Liu \\ jie Wu \\ Jianhua Jin \\ Department of Oncology, Wujin \\ People's Hospital, Affiliated to Jiangsu \\ University, Changzhou, People's \\ Republic of China
}

\begin{abstract}
Apatinib is a novel oral tyrosine kinase inhibitor targeting vascular endothelial growth factor receptor-2, which has been proved by clinical trials to be effective and safe for patients with chemotherapy-refractory gastric cancer. To date, there is no study or case report on apatinib treatment for patients with ovarian cancer. Here, we present the case of a 50-year-old Chinese woman with advanced ovarian cancer, who received apatinib at a daily dose of $500 \mathrm{mg}$ for 28 days per cycle after failure of fourth-line chemotherapy. Favorable oncologic outcome was achieved in this case after treatment with apatinib. The patient's progression-free survival is now 11.3 months, and she is taking apatinib and capecitabine as maintenance treatment. The common side effect of apatinib was fatigue; however, the toxicity of apatinib was controllable and tolerable. Thus, apatinib may be an option for chemotherapy-refractory advanced epithelial ovarian cancer, but this still warrants further investigation.
\end{abstract}

Keywords: advanced epithelial ovarian cancer, apatinib, chemotherapy, angiogenesis inhibitor, targeted therapy

\section{Introduction}

Ovarian cancer is the eighth leading cause of cancer death among women, with 238,700 new cases and 151,900 deaths in 2012 worldwide. ${ }^{1}$ For most patients with AEOC, the first-line treatment consists of a debulking surgery and chemotherapy with platinum combined with paclitaxel or docetaxel., ${ }^{2,3}$ Despite significant initial response to platinum-based chemotherapies, many patients relapse. However, no standard chemotherapy regimen (beyond the second-line) has been established.

Angiogenesis is a target in the treatment of ovarian cancer, and antiangiogenic drugs such as BV have been actively investigated in the adjuvant setting and recurrent ovarian cancer. ${ }^{4-8}$ However, not everyone can afford the high cost of BV treatment.

Apatinib (Hengrui Pharmaceutical Co., Ltd., Shanghai, People's Republic of China) is a small-molecule TKI targeting VEGFR-2, which has been proved to be effective and safe in treating chemotherapy-refractory advanced gastric cancer. ${ }^{9,10}$ Recently, apatinib is more commonly used in clinical practice for metastatic breast cancer, esophageal cancer, non-small-cell lung cancer, and hepatocellular carcinoma. However, there is no report available on its efficacy and safety in patients with ovarian cancer. Herein, we present the case of a female patient with AEOC treated using apatinib in our hospital.

\section{Case report}

A 50-year-old female with AEOC was admitted to Wujin People's Hospital in Changzhou on October 25, 2015, for abnormal vaginal discharge. The patient received a 
debulking surgery on April 25, 2012, including total hysterectomy, bilateral adnexectomy, greater omentum resection, and appendectomy. Intraoperative, miliary lesions were found in the leaves of the diaphragm, the liver, the peritoneum, and the surface of the intestine. The patient's serum CA125 level was 1,317.00 U/mL on April 17, 2012, preoperatively, and then decreased to 459.20 U/mL on May 7, 2012, postoperatively. The pathology was confirmed as papillary serous cystadenocarcinoma in both the ovaries with metastatic implants in the omentum. Immunohistochemical analysis showed that the patient was CK7(+), ER(+/-), PR(+), E-Cad(+), P53(-), and Vim(-). She was staged as FIGO 2010 Stage IIIC according to the pathology report. However, 10 days after the surgery, the patient underwent abdominal CT which revealed several sporadic metastases in the liver, and therefore, she was restaged as FIGO 2010 Stage IV and was treated with postoperative intravenous paclitaxel $\left(135 \mathrm{mg} / \mathrm{m}^{2}\right)$ and cisplatin $\left(75 \mathrm{mg} / \mathrm{m}^{2}\right)$ for six cycles. Her serum CA125 level was within the normal range.

The patient did well until February 2014, after which her serum CA125 level was found to be increased to $88.14 \mathrm{U} / \mathrm{mL}$ and CT scan showed a metastatic mass in front of the rectum. She was treated with paclitaxel and cisplatin as second-line chemotherapy for six cycles again, during which the serum CA125 level decreased and CT scan indicated SD according to the RECIST 1.0.

In February 2015, 7 months after the end of second-line chemotherapy, the patient had intermittent vaginal bleeding, and her serum CA125 level was $388.80 \mathrm{U} / \mathrm{mL}$. The chest and abdominal CT scan indicated that compared with the previous evaluation, the mass in front of the rectum has grown bigger and revealed a new metastasis lymph node in the left side of the pelvic cavity. Following this, the patient was treated with gemcitabine $\left(1,000 \mathrm{mg} / \mathrm{m}^{2}\right.$, on days 1 and 8$)$ and oxaliplatin (75 mg/m², on days 1 and 8) as third-line chemotherapy, which was repeated every 3 weeks. After two cycles of chemotherapy, the CT scan indicated SD, but the CA125 level was increased to $490.20 \mathrm{U} / \mathrm{mL}$ and the patient experienced anal pendant expansion. Hence, she was switched to receive four cycles of fourth-line chemotherapy consisting of pemetrexed $500 \mathrm{mg} / \mathrm{m}^{2}$ on day 1 , and cisplatin $25 \mathrm{mg} / \mathrm{m}^{2}$ on days $1-3$, which was repeated every 3 weeks. Intermittent vaginal bleeding and anal pendant expansion were significantly relieved after two cycles of chemotherapy. The therapeutic evaluation indicated SD, and CA125 level was decreased to $291.9 \mathrm{U} / \mathrm{mL}$ after four cycles of chemotherapy.

In October 2015, 2 months after the end of fourth-line chemotherapy, the patient reported abnormal vaginal discharge, and her serum CA125 level was 494.70 U/mL. The CT scan showed that compared with the previous evaluation (August 17, 2015; Figures 1A and 2A), both the lymph node in the left side of the pelvic cavity (October 22, 2015; Figure 1B) and the mass in front of the rectum (October 22, 2015; Figure 2B) were bigger. Then, she received epirubicin $80 \mathrm{mg} / \mathrm{m}^{2}$ and cyclophosphamide $600 \mathrm{mg} / \mathrm{m}^{2}$ as fifth-line chemotherapy. After one cycle, her serum CA125 level rose to $543.70 \mathrm{U} / \mathrm{mL}$ on November 19 , 2015. Therefore, we recommended her to try chemotherapy combined with apatinib for antiangiogenesis therapy. The patient signed the informed consent for administering apatinib, which was approved by the Medical Ethics Committee of Wujin People's Hospital. The patient also provided her written informed consent for the accompanying images to be published in this case study. She was hospitalized, and her ECOG performance status was 1. She received EC chemotherapy on November 22, 2015 , and was orally administered apatinib (500 mg/d) since November 23, 2015. One month later, her serum CA125 level decreased to $327.80 \mathrm{U} / \mathrm{mL}$, and the abnormal vaginal discharge was reduced too. Therefore, she continued to receive EC chemotherapy on December 25, 2015, combined with apatinib. Due to the Chinese Spring Festival, she stopped chemotherapy for 2 months, and only took apatinib orally. Two months later, the CT scan (March 7, 2016; Figures 1C and 2C) indicated SD, and the serum CA125 level was decreased to $160.50 \mathrm{U} / \mathrm{mL}$. Then, she continued to receive three cycles of EC chemotherapy from March 9, 2016, to May 11, 2016, combined with apatinib. During this period, her serum CA125 level continually reduced, and the abnormal vaginal discharge disappeared. After totally six cycles of EC chemotherapy, she refused to receive intravenous chemotherapy again. The serum CA125 level was found to be decreased to $93.47 \mathrm{U} / \mathrm{mL}$ on June 6 , 2016. According to the single-agent therapies mentioned in the NCCN Guidelines for ovarian cancer (version 2, 2015), we have recommended her to take capecitabine orally combined with apatinib for maintenance treatment until now. Capecitabine was administered at a dose of $1,000 \mathrm{mg} / \mathrm{m}^{2}$ orally bid with meals for 14 days on a 21-day cycle from June 6, 2016. The latest CT scan (August 2016; Figures 1D and 2D) indicated SD, and the serum CA125 level was still falling (Figure 3). The PFS of the patient since treatment with apatinib is 11.3 months now. During the treatment of apatinib, the patient experienced Grade 2 fatigue and it was well managed. No hypertension, hand-foot skin reaction, or proteinuria occurred. 

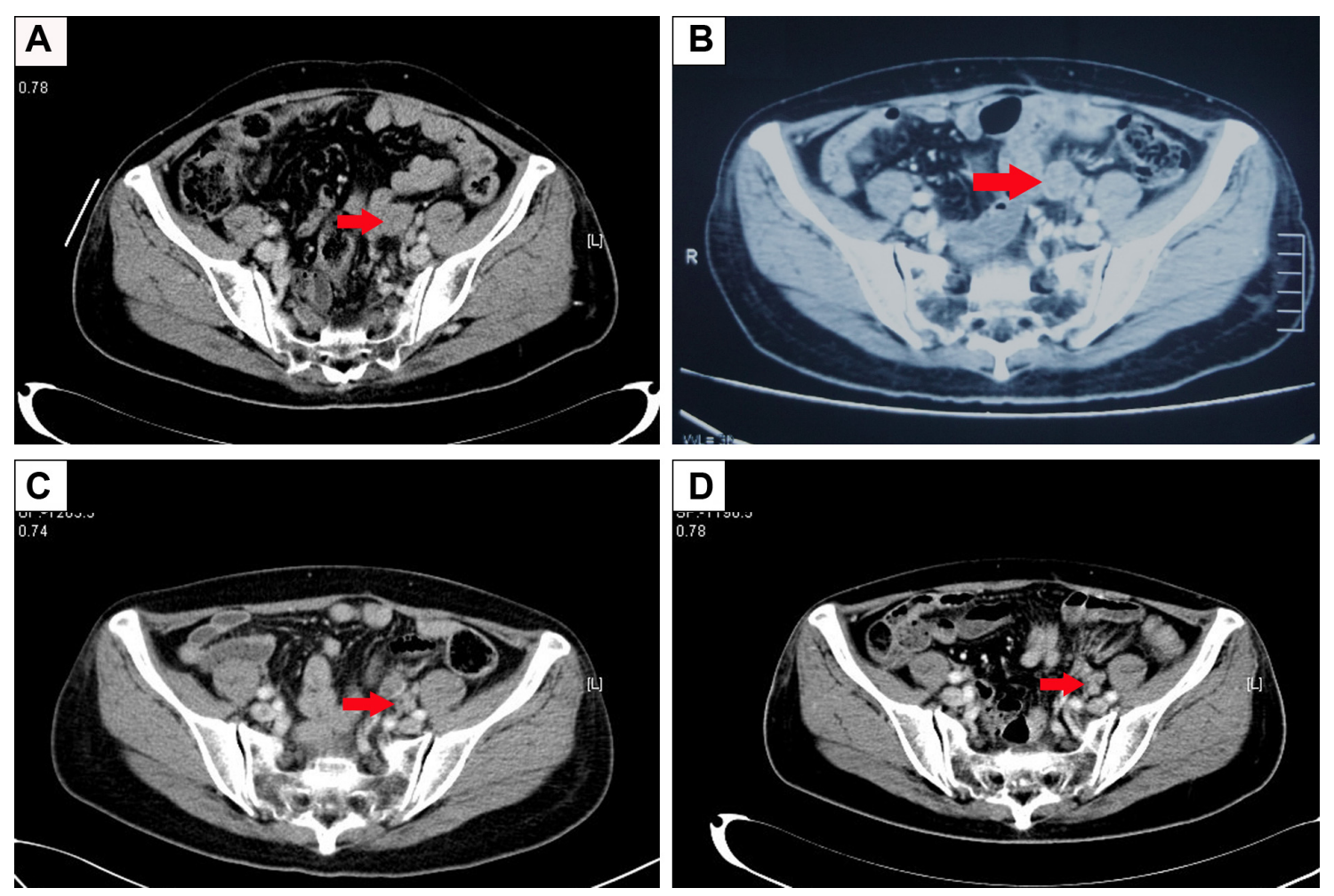

Figure I Pelvic CT shows the lymph node metastasis. (A) Before therapy (August 17, 2015). (B) Before therapy (October 22, 2015), the lymph node was bigger than 2 months earlier. (C) After 3 months of apatinib treatment (March 7, 2016), the lymph node was smaller than 4 months earlier. (D) After 9 months of apatinib treatment (August 30, 2016), the lymph node was smaller than 5 months earlier. Red arrows indicate the lymph node metastasis.

Abbreviation: $\mathrm{CT}$, computed tomography.
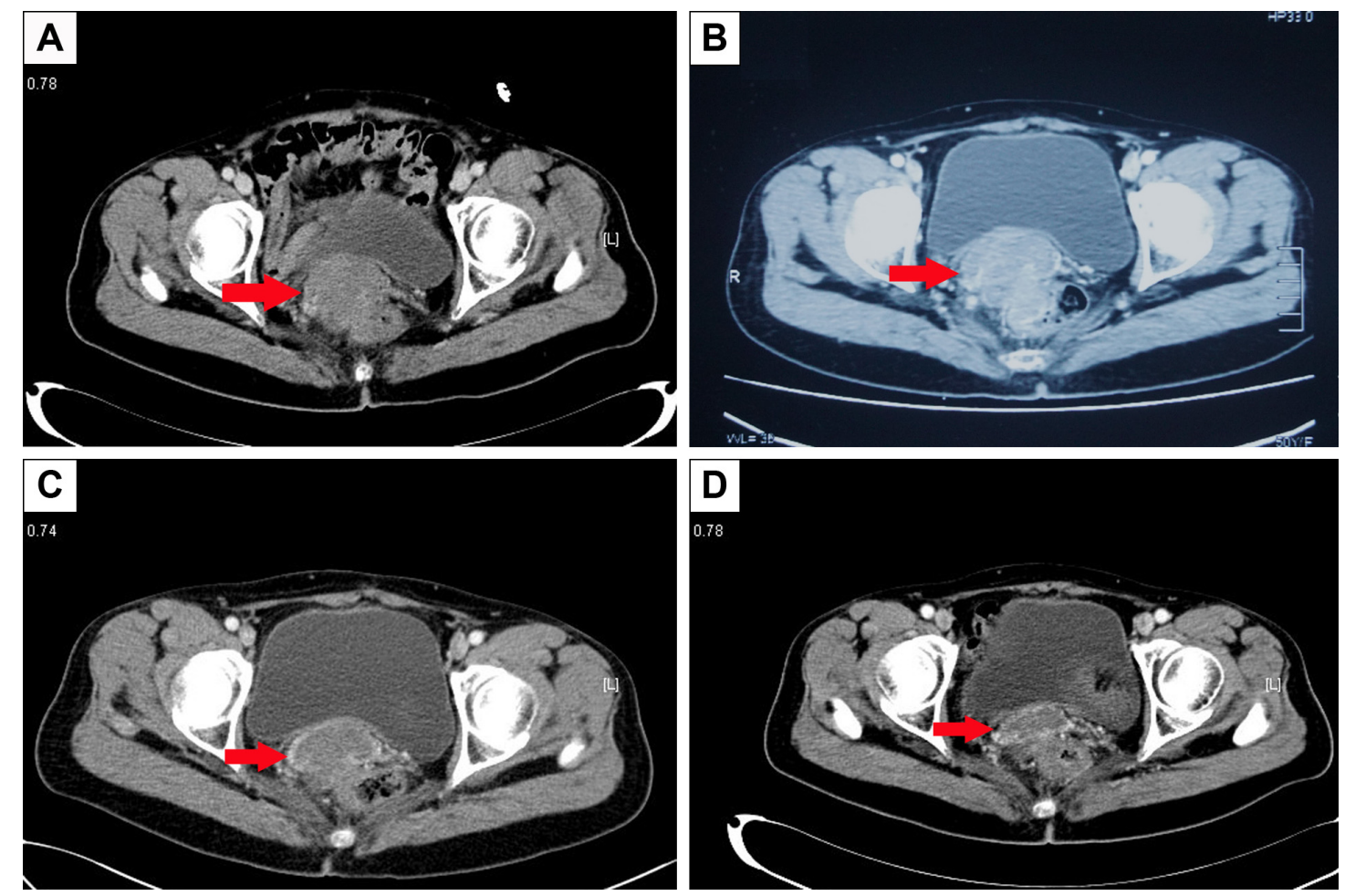

Figure 2 Pelvic CT shows the metastatic mass in front of the rectum. (A) Before therapy (August 17, 2015). (B) Before therapy (October 22, 2015), the mass was bigger than 2 months earlier (C) After 3 months of apatinib treatment (March 7, 2016), the mass was smaller than 4 months earlier. (D) After 9 months of apatinib treatment (August 30 , 2016), the mass was smaller than 5 months earlier. Red arrows indicate the metastatic mass in front of the rectum.

Abbreviation: $\mathrm{CT}$, computed tomography. 


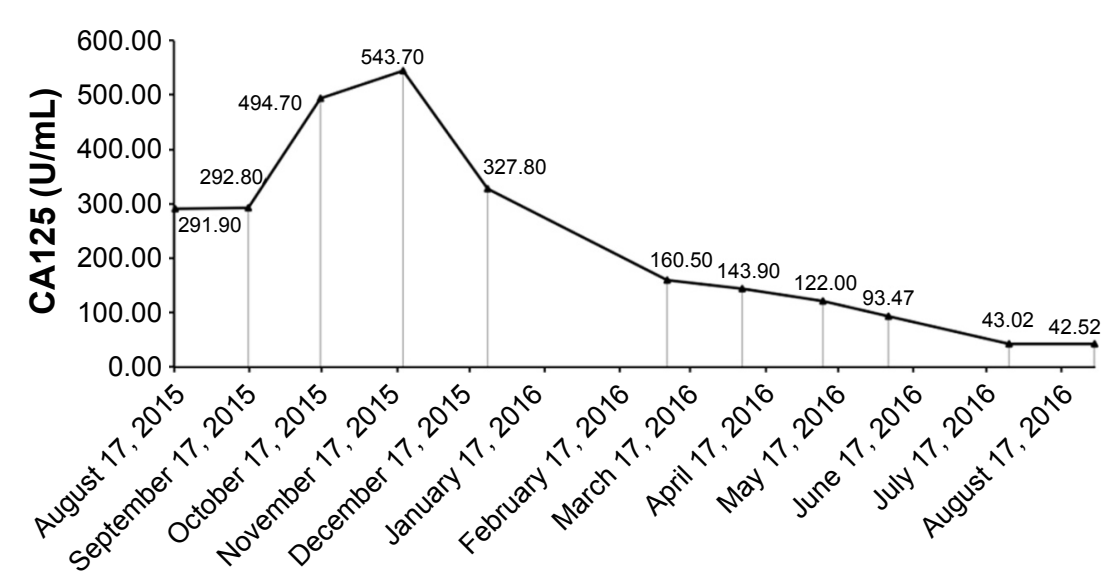

Date

Figure 3 The serum CAI25 level during the treatment with apatinib.

Abbreviation: CAI25, cancer antigen 125.

\section{Discussion}

To the best of our knowledge, this is the first case report on apatinib treatment for AEOC.

Apatinib, also known as YN968D1, is one of the latest oral small-molecule TKIs that inhibits VEGFR-2 (Flk-1/ $\mathrm{KDR}$ ), RET, c-Kit (stem cell factor receptor), and c-Src tyrosine kinases. ${ }^{11}$ In a Phase II randomized clinical trial, 144 patients with advanced metastatic gastric cancer who had failed two or more lines of chemotherapy were enrolled. The patients were randomized into PL group or two apatinib groups which received $850 \mathrm{mg}$ once daily or $425 \mathrm{mg}$ twice daily. Median OS of the three groups was 2.50 months, 4.83 months, and 4.27 months, respectively. Median PFS was 1.40 months, 3.67 months, and 3.20 months, respectively. DCRs were $11.63 \%, 58.54 \%$, and $40 \%$, respectively. Significant differences were found in OS, PFS, and DCR between the apatinib and PL groups. ${ }^{9}$ In another Phase III randomized, double-blind multicenter trial in the People's Republic of China, 267 patients with advanced or metastatic adenocarcinoma of the stomach or gastroesophageal junction who had failed two or more prior lines of chemotherapy were enrolled. The findings of the trial showed that both the median OS and median PFS were significantly improved in the apatinib group compared with the PL group (6.5 months vs 4.7 months $[P=0.0149]$ and 2.6 months vs 1.8 months $[P<0.001]$, respectively). Also, the DCR was significantly higher in the apatinib group than the PL group (42.05\% vs $9.19 \%, P<0.001) .{ }^{10}$ Based on these two clinical trials, apatinib was recommended for the treatment of advanced gastric adenocarcinoma and adenocarcinoma of the gastroesophageal junction by the Food and Drug Administration of the People's Republic of China.
We used apatinib combined with chemotherapy based on its efficacy in antiangiogenesis. Moreover, it is cheaper and easier to administer compared with intravenous BV. Interestingly, although the therapeutic evaluation indicated $\mathrm{SD}$, the serum CA125 level is still decreasing, and the PFS is 11.3 months now.

In the OCEANS trial, a randomized, double-blind, Phase III trial, 484 patients were randomly assigned to $\mathrm{BV}$ with $\mathrm{GC}$ or PL with GC. Compared with PL plus GC arm, the PFS of BV plus GC arm was higher (8.4 months vs 12.4 months, respectively). ${ }^{6}$ In the ICON6 trial, another randomized, double-blind, Phase III antiangiogenic trial in recurrent platinumsensitive ovarian cancer, 486 women were randomly assigned to arm A to receive PL alongside chemotherapy and then PL maintenance, arm B to receive cediranib $20 \mathrm{mg}$ once daily alongside chemotherapy and then PL maintenance, or arm $\mathrm{C}$ to receive cediranib $20 \mathrm{mg}$ once daily alongside chemotherapy and then cediranib maintenance. The PFS of arms A, $\mathrm{B}$, and $\mathrm{C}$ was 9.1 months, 9.9 months, and 11.0 months, respectively. ${ }^{12}$ What is noteworthy is that this patient received apatinib combined with chemotherapy as fifth-line therapy, and her PFS is 11.3 months now. This is similar to the BV arm in the OCEANS trial and the arm C in the ICON6 trial which received chemotherapy as second-line therapy. This indicates that apatinib may have a similar effect on ovarian cancer as BV and cediranib. Certainly, the final PFS of this case is expected, and clinical trials are required to evaluate the efficacy and tolerance in ovarian cancer.

Hypertension, hand-foot skin reaction, and proteinuria are the most common adverse effects of antiangiogenic agents; ${ }^{9,10}$ however, these are controllable and tolerable. Although, a case report showed gastrointestinal hemorrhage 
and perforation during apatinib treatment of gastric cancer. ${ }^{13}$ In our case, the patient did not experience those five side effects but Grade 2 fatigue which was well managed. The difference might have been caused by the dose of apatinib.

From this case report, three questions arise: 1) Can the combination of apatinib and chemotherapy improve the benefit in other AEOC patients? 2) What is the appropriate dose of apatinib for AEOC patients? 3) When is the best time to use apatinib in AEOC patients? To answer these questions, more clinical studies are needed; and furthermore, these questions can offer guidelines for clinical treatment.

\section{Abbreviations}

AEOC, advanced epithelial ovarian cancer; BV, bevacizumab; CA125, cancer antigen 125; CT, computed tomography; DCR, disease control rate; EC, epirubicin and cyclophosphamide; ECOG, Eastern Cooperative Oncology Group; FIGO, International Federation of Gynecology and Obstetrics; GC, gemcitabine and carboplatin; ICON6, cediranib in patients with relapsed platinum-sensitive ovarian cancer; NCCN, National Comprehensive Cancer Network; OCEANS, Ovarian Cancer Study Comparing Efficacy and Safety of Chemotherapy and Anti-Angiogenic Therapy in Platinum-Sensitive Recurrent Disease; OS, overall survival; PFS, progression-free survival; PL, placebo; RECIST 1.0, response evaluation criteria in solid tumors version 1.0; $\mathrm{SD}$, stable disease; TKI, tyrosine kinase inhibitor; VEGFR, vascular endothelial growth factor receptor.

\section{Acknowledgments}

The authors thank the patient for her participation and her agreement to publication of the report. This work was supported by Changzhou Wujin Sci \&Tech Program (WS201505).

\section{Author contributions}

All authors contributed toward data analysis, and drafting and critically revising the paper, gave final approval of the version to be published, and agreed to be accountable for all aspects of the work.

OncoTargets and Therapy

\section{Publish your work in this journal}

OncoTargets and Therapy is an international, peer-reviewed, open access journal focusing on the pathological basis of all cancers, potential targets for therapy and treatment protocols employed to improve the management of cancer patients. The journal also focuses on the impact of management programs and new therapeutic agents and protocols on

\section{Disclosure}

The authors report no conflicts of interest in this work.

\section{References}

1. Torre LA, Bray F, Siegel RL, Ferlay J, Lortet-Tieulent J, Jemal A. Global cancer statistics, 2012. CA Cancer J Clin. 2015;65(2):87-108.

2. Stuart GC, Kitchener H, Bacon M, et al; participants of 4th Ovarian Cancer Consensus Conference (OCCC); Gynecologic Cancer Intergroup. 2010 Gynecologic Cancer InterGroup (GCIG) consensus statement on clinical trials in ovarian cancer: report from the Fourth Ovarian Cancer Consensus Conference. Int J Gynecol Cancer. 2011;21(4):750-755.

3. Jayson GC, Kohn EC, Kitchener HC, Ledermann JA. Ovarian cancer. Lancet. 2014;384(9951):1376-1388.

4. Perren TJ, Swart AM, Pfisterer J, et al; ICON7 Investigators. A phase 3 trial of bevacizumab in ovarian cancer. N Engl J Med. 2011;365(26): 2484-2496.

5. Burger RA, Brady MF, Bookman MA, et al; Gynecologic Oncology Group. Incorporation of bevacizumab in the primary treatment of ovarian cancer. N Engl J Med. 2011;365(26):2473-2483.

6. Aghajanian C, Blank SV, Goff BA, et al. OCEANS: a randomized, double-blind, placebo-controlled phase III trial of chemotherapy with or without bevacizumab in patients with platinum-sensitive recurrent epithelial ovarian, primary peritoneal, or fallopian tube cancer. J Clin Oncol. 2012;30(17):2039-2045.

7. Pujade-Lauraine E, Hilpert F, Weber B, et al. Bevacizumab combined with chemotherapy for platinum-resistant recurrent ovarian cancer: the AURELIA open-label randomized phase III trial. J Clin Oncol. 2014; 32(13):1302-1308.

8. Oza AM, Cook AD, Pfisterer J, et al; ICON7 trial investigators. Standard chemotherapy with or without bevacizumab for women with newly diagnosed ovarian cancer (ICON7): overall survival results of a phase 3 randomised trial. Lancet Oncol. 2015;16(8):928-936.

9. Li J, Qin S, Xu J, et al. Apatinib for chemotherapy-refractory advanced metastatic gastric cancer: results from a randomized, placebo-controlled, parallel-arm, phase II trial. J Clin Oncol. 2013;31(26):3219-3225.

10. Li J, Qin S, Xu J, et al. Randomized, double-blind, placebo-controlled phase III trial of apatinib in patients with chemotherapy-refractory advanced or metastatic adenocarcinoma of the stomach or gastroesophageal junction. J Clin Oncol. 2016;34(13):1448-1454.

11. Mi YJ, Liang YJ, Huang HB, et al. Apatinib (YN968D1) reverses multidrug resistance by inhibiting the efflux function of multiple ATPbinding cassette transporters. Cancer Res. 2010;70(20):7981-7991.

12. Ledermann JA, Embleton AC, Raja F, et al; ICON6 collaborators. Cediranib in patients with relapsed platinum-sensitive ovarian cancer (ICON6): a randomised, double-blind, placebo-controlled phase 3 trial. Lancet. 2016;387(10023):1066-1074.

13. Li XF, Tan YN, Cao Y, Xu JH, Zheng S, Yuan Y. A case report of gastrointestinal hemorrhage and perforation during apatinib treatment of gastric cancer. Medicine (Baltimore). 2015;94(39):e1661. patient perspectives such as quality of life, adherence and satisfaction. The manuscript management system is completely online and includes a very quick and fair peer-review system, which is all easy to use. Visit http://www.dovepress.com/testimonials.php to read real quotes from published authors. 